Journal of Emergency Primary Health Care

An International eJournal of Prehospital Care Research, Education, clinical Practice, Policy and Service Delivery ISSN 1447-4999

Original Research

\title{
TOWARDS A NATIONAL RESEARCH AGENDA FOR THE AMBULANCE AND PRE-HOSPITAL SECTOR IN AUSTRALIA
}

\author{
Vivienne Tippett, MPH - Research Fellow, Australian Centre for Pre-hospital Research \\ Michele Clark, PhD - Director, Australian Centre for Pre-hospital Research and \\ Associate Professor, School of Population Health, University of Queensland \\ Shelley Woods, MJ - Research Officer, Australian Centre for Pre-hospital Research \\ Gerry FitzGerald, MD - Chief Health Officer, Queensland Health
}

\begin{abstract}
By comparison to other health disciplines and medical specialties, pre-hospital and emergency medical systems (EMS) research lags behind its clinical cousins. This paper describes a recent Australian response to recognition of the need to improve the profile of pre-hospital and EMS research. In August 2002, the Convention of Ambulance Authorities (CAA) hosted a national symposium to discuss the development of an Australian agenda for ambulance and prehospital research. The aims of the symposium were to describe the current state of Australian research on pre-hospital care and EMS; identify gaps in the current research effort; discuss targets for future research; and describe mechanisms for encouraging industry cooperation and fostering the research effort. Similarities between the key issues facing Australian researchers and those described in both the United Kingdom and United States were noted. This Symposium was an important preliminary step in focusing and improving the prehospital research effort in Australia.
\end{abstract}

\section{Keywords: emergency care; ambulance; pre-hospital; research}

\section{Introduction}

Evidence-based practice is now considered a benchmark of best practice performance in most health disciplines. High-quality research programmes are necessary to develop the desired evidence base and have been shown to enhance the efficacy of medical care [1]. Considerable attention has been paid over the past five years to the state of research in pre-hospital care and emergency medical systems. Callaham [2] called for a serious re-examination of Emergency Medical System (EMS) research based on an assessment of the paucity of Class 1, scientific support for EMS interventions and because the monitoring of outcomes and adverse effects are considered to be poor. A more recent survey of the pre-hospital literature for the World Health Organisation [3] found only 24 randomised controlled trials and concluded that there was insufficient evidence to provide a secure evidence base for many common pre-hospital interventions. Other authors have commented more broadly on the dearth of research in prehospital care [4, 5]. In response to this, Jacobs [6] stresses the importance of multi-centre collaborative studies and data sharing to resolve the perpetual 'bugbear' of small numbers and statistical power in pre-hospital research. 
In the United States, the recently re-published National EMS Research Agenda [7] made eight key recommendations for furthering research, directly reflecting the identified impediments to conducting EMS research. The development of a critical mass of career EMS researchers and the establishment of Centres of Excellence were seen as necessary to facilitate development of a research community. Naturally, recommendations of this nature bring with them the imperatives of securing an adequate funding base to support research activity.

The American agenda recommends that Federal agencies sponsoring research should acknowledge the importance of this aspect of health research and commit appropriate resources to high-quality research. Similarly, States, corporations and charitable foundations should be encouraged to support EMS research.

In part, securing this level of engagement from funding institutions requires a coordinated, effective industry and scientific lobby. Ideally, the efforts of EMS professionals, delivery systems, academic centres and public policy makers should be organised to support and apply the results of research. Associated with this, it was recommended that EMS professionals hold themselves to higher standards of requiring evidence before implementing new procedures, drugs and devices. Development of standardised data collection methods at all levels was recommended.

There was also seen to be an evident need for the development of EMS-specific consent strategies as well as revision of existing regulations to reduce the impediments to research while continuing to adequately protect research subjects.

Cooke and Wilson [8] examined the reasons for the lack of research in pre-hospital care in the United Kingdom. On the basis of survey results from training managers in 48 ambulance services, they found that there did not appear to be any obstacles to generating topics for research nor to participation in research as a professional priority. However, lack of research training and support, together with difficulties perceived with follow-up of patient outcomes, were identified as being particular impediments. These authors called for a distinction to be made between developing the skills required for appraising and using research evidence and those required for undertaking research.

In many States, information access and integration is complex between the pre-hospital and acute health sector. In Australia, as elsewhere, the absence of a standard or core pre-hospital data set and variable access to relevant data sets outside the pre-hospital sector presents a number of difficulties to researchers, for example, the measurement of the impact of prehospital interventions on patient outcomes, such as survival to discharge.

In this context, the express aim of the national Research Symposium, held in Queensland in August 2002 and sponsored by the Convention of Ambulance Authorities, was to identify:

- the current state of Australian research on pre-hospital care and emergency medical systems (EMS);

- areas in which research should be undertaken and, where possible, prioritise targets for the future;

- ways in which pre-hospital and EMS research can be encouraged; and

- ways in which industry may cooperate to foster research. 
This paper describes this recent Australian response to recognition of the need to raise the profile of pre-hospital and EMS research.

\section{Methods}

Delegates from State and Territory ambulance authorities and retrieval services, academic units conducting pre-hospital research or involved in paramedic training, representatives of key stakeholder groups, such as the Australian College of Ambulance Professionals (ACAP) and other experts in the field, attended. Participants engaged in both small group work and plenary discussion throughout the course of the two-day meeting.

Brief presentations on the current state of research were invited from each State or Territory. Participants critiqued the strengths, weaknesses, opportunities and threats (SWOT) facing prehospital and EMS research. Small groups reported back to the plenary group at the conclusion of their deliberations. The facilitator recorded the frequency with which the same or similar opinions were expressed by each of the small groups' SWOT analysis. This activity formed the basis of the thematic analysis of the outcomes of the first day deliberations.

The second day of the Symposium began with a plenary session in which the thematic analysis of the previous day's SWOT was ratified. Participants were then briefed on the key tasks for the day, which were to:

- examine the possibilities for future pre-hospital and EMS research and discuss priorities for research;

- determine how that research could be encouraged; and

- describe their expectations for the role that Convention and other stakeholders might have in supporting a national research effort.

Following plenary determination of research areas requiring attention in the future, participants were asked to self-select into one of the four groups described by the draft model (see Figure 1) and were required to address the questions:

- How could research in this topic area be encouraged? and

- How might industry cooperate to foster this research?.

\section{Results}

Participants debated the scope and definition of the pre-hospital sector. In particular, the inclusion of pre-hospital providers other than ambulance services (e.g. aero-medical retrieval teams and first responders). In this context, the interface between the pre-hospital services and emergency departments was discussed. The significance to research of a continuum of care model and the importance of examining pre-hospital and inter-hospital care were also considered.

Thematic analysis of the results of small group deliberations demonstrated the following consistent strengths:

- Convention's commitment to research and acknowledgement of support from other peak professional bodies, such as the Australian College of Ambulance Professionals.

- Interstate commonalities and a non-competitive environment that facilitated a preparedness to be open and collaborative.

- Common tertiary educational base for paramedics.

- Enthusiasm for research. 
- Acknowledgement that pre-hospital services are valued by the community.

A review of the impediments to the performance of high-quality research identified by the national EMS research agenda for the United States published in 2002 [7] demonstrated a considerable congruence between the analysis of weaknesses in the Australian environment, specifically:

- Lack of research coordination or a national agenda, resulting in fragmentation of effort.

- Lack of identifiable EMS research funding sources.

- Ambulance officers' poor connection with research in terms of its perceived relevance to their core business.

- Lack of quality review mechanisms, publication options and dissemination strategies.

- Lack of standard data definitions and poor data quality.

- Restrictions by law on access to information.

- The perception of Ambulance services as responders rather than health agencies.

As a plenary group, participants were asked to consider whether the current emphasis of the research effort was appropriate and to identify what other research efforts should be encouraged. Participants identified a need to encourage research on:

- Workforce issues, including the development of training packages, regulation of paramedics, occupational health and safety, workforce modelling and volume projections, support and education strategies for volunteers.

- Evidence-based clinical practice, including the relationship between skill level and outcome, resuscitation methods, dissemination of clinical best practice recommendations.

- Continuum of Care issues, such as call centres and communications, first responder and community activities, intersection with acute system.

- Economics of pre-hospital care, for example cost-benefit and outcome analysis.

- Service delivery modelling and the examination of the role of ambulance services, impact of privatisation and demand for services.

- Other potential roles for ambulance services, such as health promotion and prevention activities.

- International comparison.

- Mechanisms for standardising data processes.

- Organisational performance indicators.

- Equipment evaluation.

- Target Australia's National Health Priorities (currently cardiovascular disease, diabetes, asthma, mental health and injury) [9].

- Monitoring and measurement of community expectations and consumer satisfaction.

- Managing emergencies, mass casualty incidents and biohazards. 
- Ensuring a match between "what we do, what we think we do and what patients need", or maintaining relevance.

In discussion, participants developed a draft model for grouping these areas of research into 'priority areas' (see Figure 1).

\section{FIGURE 1: RESEARCH PRIORITY MODEL}

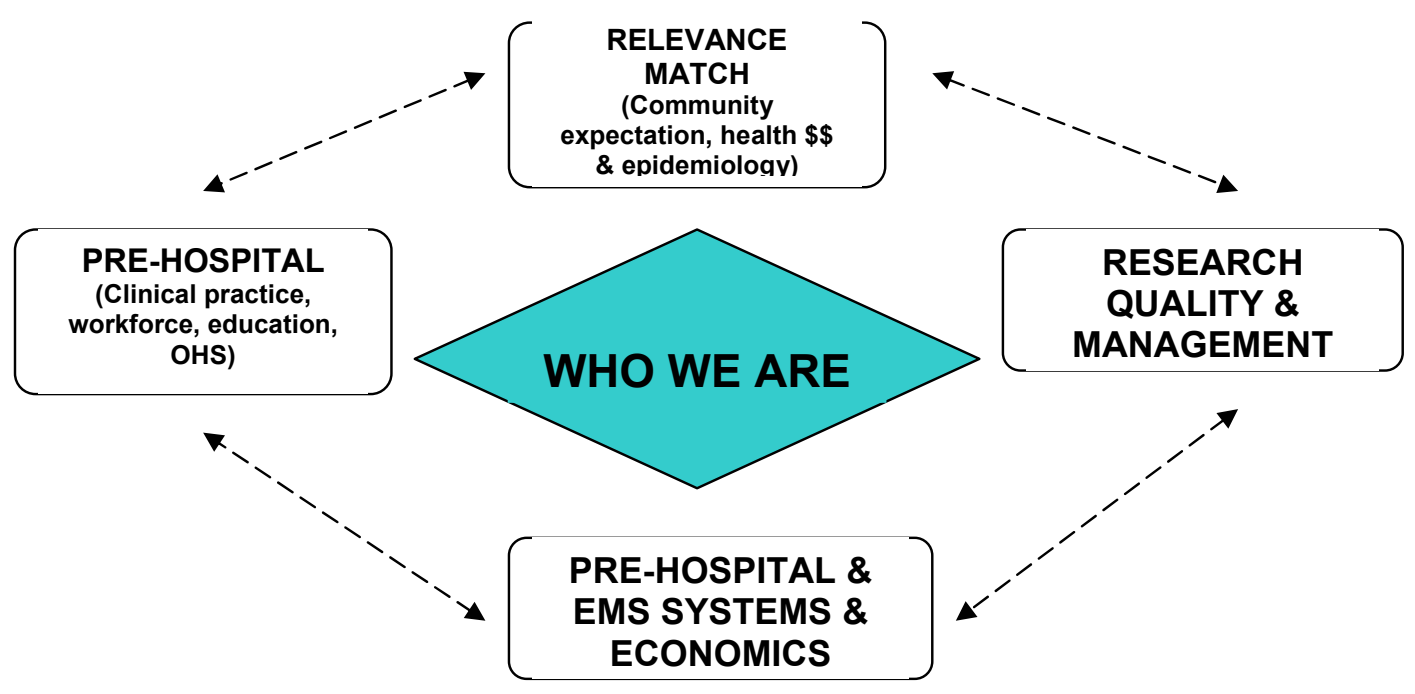

The following commentary summarises the small group deliberations on each of the four key areas.

\section{Group 1: Relevance Match}

Group 1 identified a perceived discrepancy between community need and expectation associated with ambulance services being identified as transport rather than health services. The group considered that application of primary health care models to pre-hospital and EMS research would be beneficial. The group further identified the National Health Priorities and structures such as the Public Health Partnerships [10,11] and the Rural Health Strategy [12] as potential means of ensuring the relevance of ambulance services to communities.

\section{Group 2: Research Quality and the Management of Data}

The group identified the need to determine why previous attempts at establishing a prehospital Minimum Data Set had floundered. The group also identified the need for the development of a:

- Research Sub-Committee;

- career structure to encourage research and identifying research mentors;

- a national network of EMS researchers; and

- a national collaborative dissemination strategy for the results of research effort.

The Convention of Australian Ambulance Authorities was perceived as a potential facilitator of these activities. 


\section{Group 3: Pre-hospital and EMS Systems}

The group identified the need to develop a consensus on what EMS stands for in the Australian situation and develop:

- system evaluation tools;

- means of modelling resources, the economics of pre-hospital care, equipment and communications systems;

- clinical standards; and

- quality systems improvement loops through cost-benefit analyses.

\section{Group 4: Pre-hospital/EMS Workforce}

The group identified the need for a strong recruitment strategy to ensure an appropriate workforce volume and recommended:

- a responsive education and professional development strategy for staff;

- a means of ensuring a dynamic relationship between curriculum development and best practice in clinical process;

- development of mechanisms for validating current clinical practice; and

- development of clinical outcome measures.

To achieve this, the group identified the need for:

- an industry commitment to research;

- a facilitated research culture including infrastructure (equipment and financial);a national framework for research; and

- mechanisms for encouraging 'top-down' and 'bottom-up' approaches to informing the ongoing development of research.

Participants concluded the session by discussing the next steps to facilitate development of the proposed research agenda.

\section{Discussion}

The level of congruence between the United States Agenda and the results of this symposium are noteworthy and suggest the global nature of some of the issues that warrant attention in order for research in this area to advance.

In accordance with the agreed 'next steps' following the Symposium, a working party was established. In September 2002, the group met to consider the Report on the Symposium outcomes [13] and as a result of this meeting, the national conference of the Convention of Ambulance Authorities recommended in October 2002 that while determining a national set of priorities for pre-hospital research was beyond its remit, the Symposium should be held annually to continue to encourage strategic thinking about furthering pre-hospital research.

The research activity presentations from the States and Territories at the commencement of the Symposium demonstrate a growing body of pre-hospital research in Australia. However, review of the National Health and Medical Research Council (NHMRC) Project Grants commencing in 2003, show only two projects with a pre-hospital focus. Both projects relate to the management of cardiac arrest (Bernard et al: hypothermia in cardiac arrest; Jacobs et al: efficacy of adrenaline in cardiac arrest). NHMRC Grants commencing 2003 will total more than $\$ 150$ million. Perhaps further development of the capacity of the sector to produce highquality proposals for consideration, as well as greater recognition of the National Health 
Priorities and implications for pre-hospital practice, is required before the profile of prehospital research can be raised with funding bodies of this calibre.

There are, of course, many other research funding opportunities to be explored through other Federal and State Government sources as well as corporate, industry and peak professional groups sponsorship. Funding success through these sources is equally reliant on the provision of high-calibre proposals, together with cognisance of the significance of pre-hospital research. There appears to be the need for a strong industry and academic lobby to secure acknowledgement of the importance of pre-hospital research to funding bodies.

Communication and collaboration could also be seen to be necessary platforms to maximise the value of the pre-hospital research dollar. To date, attempts to establish a national register of pre-hospital/EMS research in Australia have met with varying success. This may gain impetus in the light of the recent announcement of examination of opportunities for the establishment of an international pre-hospital Network within the Cochrane Collaboration, to be discussed at an international meeting to be held in Melbourne in early May 2003.

This Convention of Ambulance Authorities Symposium was an important preliminary step in focusing and improving the pre-hospital research effort in Australia. However, building a strong body of evidence for pre-hospital practice will require an ongoing, targeted and coordinated research effort, leadership in research excellence and a concerted lobby directed to securing recognition of the importance of pre-hospital care to patient outcomes and wider health system performance.

\section{References}

1. Brown A, Griffiss M. Effect of integrated research programs on health care systems and costs. Mil Med 1996; 161:691-5.

2. Callaham M. Quantifying the scanty science of pre-hospital emergency care. Ann Intern Med 1997 Dec; 30(6):785-90.

3. Bunn F, Kwan I, Roberts I, Wentz R. Effectiveness of pre-hospital trauma care. Report to the World Health Organisation Pre-hospital Care Steering Committee. Geneva: WHO, 2001 in: Coats TJ, Davies G. Pre-hospital care of road traffic fatalities. Br Med J 2002; 324 (May 11): 1135-8.

4. Brazier H, Murphy AW, Lynch C, Bury G. Searching for the evidence in pre-hospital care: a review of randomized controlled trials. J Accid Emerg Med 1999; 16:18-23.

5. Erich J. Ending the opinion age: seeking evidence behind EMS. Emerg Med Serv 2002 May; 45-58.

6. Jacobs I. Pre-hospital care: A plea for more research. Emerg Med 2000 12:175-6.

7. National EMS Research Agenda. PreHosp Emerg Care 2002 July/September; 6 (3 Suppl): S1-S43.

8. Cooke MW, Wilson S. Obstacles to research in pre-hospital care. Pre-hospital Immediate Care 1999 3:149-51. 
9. Wall BP, Wood LJ, Holman CDJ. Review of the National Health Priority Areas Initiative. Canberra: DHAC. 1999.

10. National Public Health Partnership. Discussion Paper on the National Public Health Partnership. September 1996. http://www.nphp.gov.au/discus.htm

11. Duckett S, Oldenberg B. National Public Health Partnership. Final Evaluation Report. La Trobe University and Queensland University of Queensland. January 2002.

12. Australian Health Ministers Conference. Healthy Horizons: A framework for improving the health of rural, regional and remote Australians 1999 - 2003. Canberra: DHAC. 1999.

13. Tippett V. (2002) Towards a Pre-hospital and Ambulance Research Agenda. Convention of Ambulance Authorities.

\section{Acknowledgements}

Convention of Ambulance Authorities

Queensland Ambulance Service

This article was peer reviewed for the Journal of Emergency Primary Health Care Vol. 1 (1-2), 2003 